\title{
Electrostatic Interaction between Two Ion-Penetrable Charged Spheroids
}

\author{
Jyh-Ping Hsu ${ }^{1}$ and Bo-Tau Liu \\ Department of Chemical Engineering, National Taiwan University, Taipei, Taiwan 10617, Republic of China
}

Received November 19, 1996; accepted March 24, 1997

\begin{abstract}
The electrostatic interaction between two ion-penetrable, charged spheroidal particles is examined theoretically. These particles can assume different sizes and an arbitrary spatial orientation. The electrical potential distribution is derived analytically under the D ebye- Huckle condition. The results for two interaction spheres, one spheroidal particle and a planar surface, and rigid particles covered by an ion-penetrable membrane can be recovered as the special cases of the present general problem. We show that, for a fixed center-to-center distance between two particles, regardless of their relative sizes, the interaction free energy is the greatest if their major axes lie on the same line (head-to-head), and the smallest if their major axes are perpendicular to each other but not on the same plane (perpendicular). $\odot 1997$ Academic Press

Key Words: electrostatic interaction, free energy; particles, spheroidal, ion-penetrable; electrical potential distribution, analytical expression.
\end{abstract}

\section{INTRODUCTION}

A dispersed system is characterized by the entity-entity and entity-medium interactions. Among these interactions the electrostatic interaction between the dispersed entities is of fundamental significance. It is prerequisite to the determination of the essential properties of the system. The classic DLVO theory, for example, is based on the electrical and the van der Waals interactions between two charged entities (1). The first step to evaluate the former is to determine the corresponding electrical potential distribution. At equilibrium, this distribution is governed by the Poisson-Boltzmann equation (1). Solving this equation for a system containing two or more charged entities can be nontrivial, in general, even under the Debye-Huckle condition (low electrical potential). The major problems needed to be resolved are the selection of a global coordinate system and the determination of the linear combination coefficients in the general solution of the governing Poisson-Boltzmann equation on

\footnotetext{
${ }^{1}$ To whom correspondence should be addressed. Fax: 886-2-3623040. E-mail: T8504009@ccms.ntu.edu.tw.
}

the basis of the boundary conditions specified. For an arbitrary geometry and boundary conditions, this is not an easy task. Reported results for two interacting charged entities in an electrolyte solution are limited in the literature. Ohshima and Kondo (2) considered the electrical interactions between two charged, ion-penetrable spheres. They showed that if the fixed charges in the spheres are distributed homogeneously, both the electrical potential distribution and the interaction energy can be derived analytically. The analysis was also extended to the case of an ion-penetrable sphere and a rigid surface (3). An image method was adopted which applies the result for the case of two ion-penetrable spheres. Some other results for two spherical particles include Ohshima (4-8), Ohshima and Kondo (9), Krozel and Saville (10), and Sengupta and Papadopoulos (11). Allain and Cloitre (12) examined the interaction between two parallel cylinders. The electrical interaction between two twodimensional inclined platy particles was discussed by Anandarajah and Chen (13).

Particles having a nonrigid surface are not uncommon in practice. Biocolloids, e.g., microorganisms, can be simulated by a particle covered by an ion-penetrable membrane of finite thickness. The latter usually bears fixed charges, which arise from the dissociation of the functional groups it carries $(14,15)$. In other words, instead of spreading over a surface, the fixed charges are distributed over a finite volume in space. Another typical example is the dispersed entities in polymer-induced flocculation. In this case the polymer molecules attached to the surfaces of the dispersed entities form an adsorbed layer (16). Depending upon the types of the polymer used, this layer can carry positive, negative, or no charge.

In practice, dispersed entities may assume various shapes. Sphalerite particle, for example, is spherical, cadmium carbonate is roughly cubic, hematite is spheroidal, kaolin is planar, and alunite is polyhedral (1). Biocolloids also have several possible shapes, such as rodlike and near spherical (17). Apparently, extending the classic treatments for simple geometries to a more general case is necessary to the description of the behaviors of these particles. In the present study 


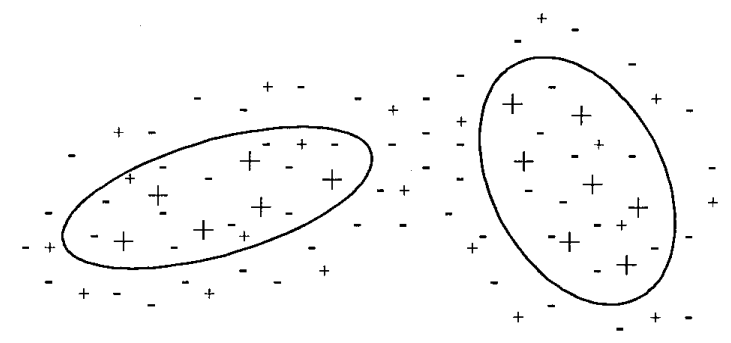

FIG. 1. A schematic representation of the problem under consideration. The particles immersed in an electrolyte solution are ion-penetrable and bear fixed charges.

the electrostatic interaction between two ion-penetrable charged entities is analyzed. We consider a family of geometries representative by spheroidal coordinates. This simulates a wide class of inorganic and biocolloids in practice, and includes most of the cases discussed in the literature.

\section{MODELING}

By referring to Fig. 1, we consider two spheroidal particles immersed in an electrolyte solution. These particles are ionpenetrable and bear fixed charges, which may due to, for example, the dissociation of the functional groups. The coordinates adopted for the problem under consideration are shown in Fig. 2, where $\{X, Y, Z\}$ and $\left\{X_{i}, Y_{i}, Z_{i}\right\}, i=1$, 2 , denote the global and the local Cartesian coordinates, respectively; $\theta_{i}$ and $\phi_{i}$ are the polar and the azimuthal angles, respectively; and $h_{i}$ is the distance between the center of particle $i$ to the global origin O. Suppose that the electrical potential of the system is sufficiently low, and the electrical potential distribution, $\psi$, can be described approximately by the linearized Poisson-Boltzmann equations

$$
\begin{gathered}
\nabla^{2} y=\kappa^{2} y, \quad \text { in solution } \\
\nabla^{2} y=\kappa^{2} y-\kappa^{2} \rho_{1}^{*}, \quad \text { inside particle } 1 \\
\nabla^{2} y=\kappa^{2} y-\kappa^{2} \rho_{2}^{*}, \quad \text { inside particle } 2,
\end{gathered}
$$

where

$$
\begin{aligned}
y & =e \psi / k_{\mathrm{B}} T \\
\kappa^{2} & =8 \pi e^{2} I / \varepsilon k_{\mathrm{B}} T \\
\rho_{i}^{*} & =\rho_{i} / 2 e I, \quad i=1,2 .
\end{aligned}
$$

In these expressions, $\rho_{i}$ is the fixed charge distribution inside particle $i, \nabla$ denotes the Laplace operator, $\psi$ is the electrical potential, $\varepsilon$ is the dielectric constant, $\kappa$ and $k_{\mathrm{B}}$ are the reciprocal Debye length and the Boltzmann constant, respectively,
$T$ is the absolute temperature, and $e$ and $I$ are, respectively, the elementary charge and the ionic strength.

The coordinates $\{X, Y, Z\}$ and $\left\{X_{i}, Y_{i}, Z_{i}\right\}$ are related by (18)

$$
\begin{aligned}
& {\left[\begin{array}{c}
X+q \cdot h_{i} \\
Y \\
Z
\end{array}\right]} \\
& =\left[\begin{array}{ccc}
\cos \theta_{i} \cos \phi_{i} & -\sin \theta_{i} & -\cos \theta_{i} \sin \phi_{i} \\
\sin \theta_{i} \cos \phi_{i} & \cos \theta_{i} & -\sin \theta_{i} \sin \phi_{i} \\
\sin \phi_{i} & 0 & \cos \phi_{i}
\end{array}\right]\left[\begin{array}{l}
X_{i} \\
Y_{i} \\
Z_{i}
\end{array}\right] \\
& =\left[\begin{array}{c}
X_{i} \\
Y_{i} \\
Z_{i}
\end{array}\right], i=1,2,
\end{aligned}
$$

where $q=1$ for particle $1(i=1)$ and -1 for particle $2(i$ $=2$ ). Since $\left[\Lambda_{i}\right]$ is an orthogonal matrix, we have (19)

$$
\left[\begin{array}{c}
X_{i} \\
Y_{i} \\
Z_{i}
\end{array}\right]=\left[\Lambda_{i}\right]^{\mathrm{T}}\left[\begin{array}{c}
X+q \cdot h_{i} \\
Y \\
Z
\end{array}\right],
$$

where the superscript $\mathrm{T}$ denotes matrix transpose. The local Cartesian coordinates $\left\{X_{i}, Y_{i}, Z_{i}\right\}$ can be transformed to the local spheroidal coordinates $\left\{\xi_{i}, \eta_{i}, \Phi_{i}\right\}$, shown in Fig. 3 , through the relations (20)

$$
\begin{aligned}
X_{i} & =a_{i} \eta_{i} \xi_{i} \\
Y_{i} & =a_{i}\left[\left(1-\eta_{i}^{2}\right)\left(\xi_{i}^{2}-j\right)\right]^{1 / 2} \cos \Phi_{i} \\
Z_{i} & =a_{i}\left[\left(1-\eta_{i}^{2}\right)\left(\xi_{i}^{2}-j\right)\right]^{1 / 2} \sin \Phi_{i}
\end{aligned}
$$

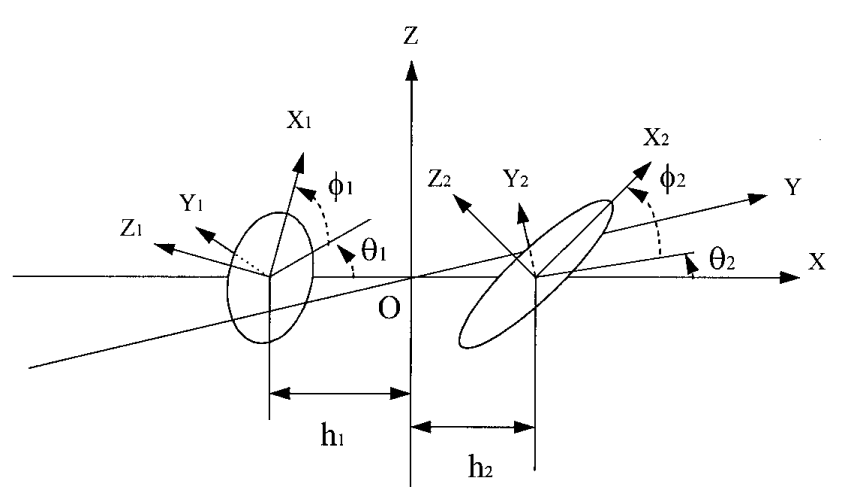

FIG. 2. The coordinate system adopted. $\{X, Y, Z\}$ and $\left\{X_{i}, Y_{i}, Z_{i}, i\right.$ $=1,2\}$, denote the global and the local Cartesian coordinates, respectively; $\theta$ and $\phi$ are the polar and the azimuthal angles, respectively; and $h_{i}$ is the distance between the center of particle $i$ to the global origin 0 . 

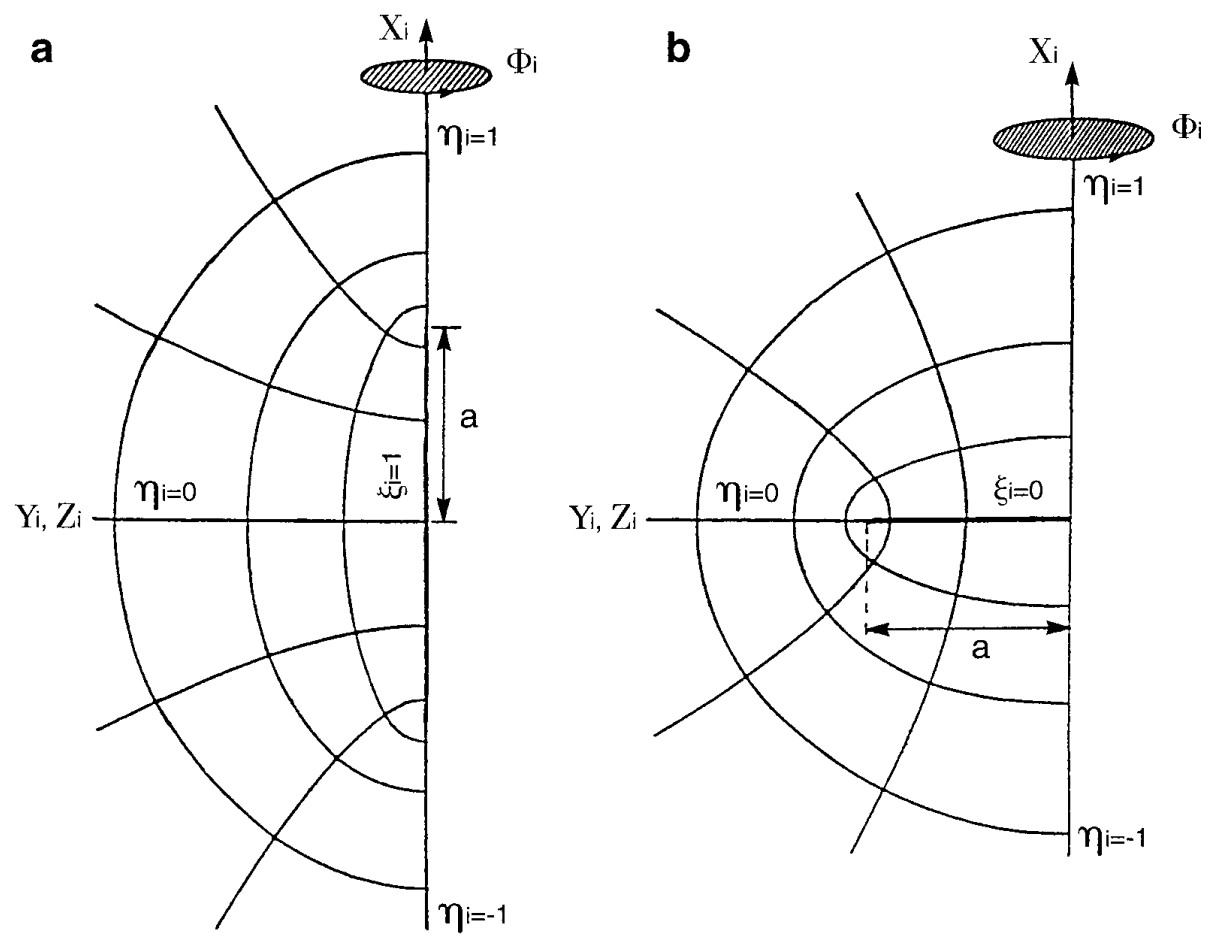

FIG. 3. The local spheroidal coordinates adopted for particle $i$. (a) Prolate spheroid; (b) Oblate spheroid.

and

$$
\begin{aligned}
\frac{X_{i}^{2}}{a_{i}^{2} \xi_{i}^{2}}+\frac{Y_{i}^{2}}{a_{i}^{2}\left(\xi_{i}^{2}-j\right)}+\frac{Z_{i}^{2}}{a_{i}^{2}\left(\xi_{i}^{2}-j\right)}=1 \\
\frac{X_{i}^{2}}{a_{i}^{2} \eta_{i}^{2}}-\frac{Y_{i}^{2}}{a_{i}^{2}\left(1-\eta_{i}^{2}\right)}-\frac{Z_{i}^{2}}{a_{i}^{2}\left(1-\eta_{i}^{2}\right)}=j \\
\tan \Phi_{i}=\frac{Z_{i}}{Y_{i}} .
\end{aligned}
$$

In these relations, $j=1$ for a prolate spheroid, $j=-1$ for an oblate spheroid, and $a_{i}$ is the distance between the focus and the center of particle $i ; \xi_{i}, \eta_{i}$, and $\Phi_{i}$ denote, respectively, the radial, the angle, and the rotation coordinates. In the case where the electrical potential is independent of $\Phi_{i}$, Eqs. [8a]- [8c] can be simplified as

$$
\begin{aligned}
& \xi_{i}=\sqrt{W+\sqrt{W^{2}-4 j\left(\frac{X_{i}}{a_{i}}\right)^{2}}} /_{2} \\
& \eta_{i}= \pm \sqrt{W-\sqrt{W^{2}-4 j\left(\frac{X_{i}}{a_{i}}\right)^{2}}} /_{2 j},
\end{aligned}
$$

where

$$
W=\left(\frac{x_{i}}{a_{i}}\right)^{2}+\left(\frac{y_{i}}{a_{i}}\right)^{2}+j .
$$

For simplicity, we assume that both the electrical potential and its derivative are continuous over the particle-liquid interface, and $\varepsilon$ is constant. In this case, the boundary conditions associated with Eqs. [1]-[3] are

$$
\begin{gathered}
y_{\xi=\xi_{\mathrm{S} i}}=y_{\xi=\xi_{\mathrm{S} i}^{+}}, \quad i=1,2 \\
\left(\frac{\partial y}{\partial \xi}\right)_{\xi=\xi_{\mathrm{S} i}^{-}}=\left(\frac{\partial y}{\partial \xi}\right)_{\xi=\xi_{\mathrm{S} i}^{+}}, \quad i=1,2,
\end{gathered}
$$

where $\xi_{\mathrm{S} i}$ denotes the $i$ th particle-liquid interface. Note that these expressions imply that the electrical potential distribution can be expressed as a linear combination of the unperturbed electrical potential distributions of each individual particle. If the distribution of fixed charge inside a particle is uniform, the unperturbed electrical potential distribution for particle $i$ is governed by

$$
\begin{aligned}
\frac{\partial}{\partial \eta_{i}}\left[\left(1-\eta_{i}^{2}\right) \frac{\partial y_{i}}{\partial \eta_{i}}\right]+\frac{\partial}{\partial \xi_{i}} & {\left[\left(\xi_{i}^{2}-j\right) \frac{\partial y_{i}}{\partial \xi_{i}}\right] } \\
& -c_{i}^{2}\left(\xi_{i}^{2}-j \eta_{i}^{2}\right) y_{i}=0,
\end{aligned}
$$

where

$$
c_{i}=\kappa a_{i} .
$$


The solution to Eq. [11a] subject to Eqs. [10a] and [10b] can be expressed as a linear combination of the spheroidal wave functions (Appendix) as

$$
\begin{aligned}
& y_{i}= \sum_{m=0}^{\infty} A_{2 m}\left(c_{i}\right) R_{2 m}^{(2)}\left(c_{i}, \xi_{i}\right) S_{2 m}\left(c_{i}, \eta_{i}\right), \quad \xi_{\mathrm{S} i} \leqslant \xi_{i} \\
& y_{i}=\rho_{i}^{*}+\sum_{m=0}^{\infty} B_{2 m}\left(c_{i}\right) \\
& \quad \times\left(R_{2 m}^{(1)}\left(c_{i}, \xi_{i}\right)-\frac{R_{2 m}^{\prime(1)}\left(c_{i}, 1\right)}{R_{2 m}^{\prime(2)}\left(c_{i}, 1\right)} R_{2 m}^{(2)}\left(c_{i}, \xi_{i}\right)\right) \\
& \quad \times S_{2 m}\left(c_{i}, \eta_{i}\right), \quad 1 \leqslant \xi_{i} \leqslant \xi_{\mathrm{S} i},
\end{aligned}
$$

where

$$
\begin{gathered}
A_{2 m}\left(c_{i}\right)=-D_{2 m} \cdot\left(R_{2 m}^{\prime(1)}\left(c_{i}, \xi_{\mathrm{S} i}\right)\right. \\
\left.-\frac{R_{2 m}^{\prime(1)}\left(c_{i}, 1\right)}{R_{2 m}^{\prime(2)}\left(c_{i}, 1\right)} R_{2 m}^{\prime(2)}\left(c_{i}, \xi_{\mathrm{S} i}\right)\right) / \operatorname{det} C_{i}, \\
B_{2 m}\left(c_{i}\right)=-D_{2 m} \cdot R_{2 m}^{\prime(2)}\left(c_{i}, \xi_{\mathrm{S} i}\right) / \operatorname{det} C_{i}, \\
D_{2 m}=\frac{\rho_{i}^{*} A_{2 m, 0}}{\sum_{w=0}^{\infty}\left[\left(A_{2 m, 2 w}\right)^{2} / 4 w+1\right]}
\end{gathered}
$$

Therefore the general solution to Eqs. [1] - [3] can be expressed as

$$
\begin{aligned}
& y=\rho_{1}^{*}+\sum_{m=0}^{\infty} B_{2 m}\left(c_{1}\right) \\
& \times\left(R_{2 m}^{(1)}\left(c_{1}, \xi_{1}\right)-\frac{R_{2 m}^{\prime(1)}\left(c_{1}, 1\right)}{R_{2 m}^{\prime(2)}\left(c_{1}, 1\right)} R_{2 m}^{(2)}\left(c_{1}, \xi_{1}\right)\right) S_{2 m}\left(c_{1}, \eta_{1}\right) \\
& +\sum_{m=0}^{\infty} A_{2 m}\left(c_{2}\right) R_{2 m}^{(2)}\left(c_{2}, \xi_{2}\right) S_{2 m}\left(c_{2}, \eta_{2}\right), 1 \leqslant \xi_{1} \leqslant \xi_{\mathrm{S} 1}, \\
& y=\sum_{m=0}^{\infty} B_{2 m}\left(c_{1}\right) R_{2 m}^{(2)}\left(c_{1}, \xi_{1}\right) S_{2 m}\left(c_{1}, \eta_{1}\right) \\
& +\sum_{m=0}^{\infty} A_{2 m}\left(c_{2}\right) R_{2 m}^{(2)}\left(c_{2}, \xi_{2}\right) S_{2 m}\left(c_{2}, \eta_{2}\right), \\
& \xi_{1} \geqslant \xi_{\mathrm{s} 1}, \xi_{1} \geqslant \xi_{\mathrm{s} 1}, \\
& y=\sum_{m=0}^{\infty} B_{2 m}\left(c_{1}\right) R_{2 m}^{(2)}\left(c_{1}, \xi_{1}\right) S_{2 m}\left(c_{1}, \eta_{1}\right)+\rho_{2}^{*} \\
& +\sum_{m=0}^{\infty} A_{2 m}\left(c_{2}\right)\left(R_{2 m}^{(1)}\left(c_{2}, \xi_{2}\right)-\frac{R_{2 m}^{\prime(1)}\left(c_{2}, 1\right)}{R_{2 m}^{\prime(2)}\left(c_{2}, 1\right)} R_{2 m}^{(2)}\left(c_{2}, \xi_{2}\right)\right) \\
& S_{2 m}\left(c_{2}, \eta_{2}\right), 1 \leqslant \xi_{2} \leqslant \xi_{\mathrm{S} 2} .
\end{aligned}
$$

The relation between $\left\{\xi_{1}, \eta_{1}, \Phi_{1}\right\}$ and $\left\{\xi_{2}, \eta_{2}, \Phi_{2}\right\}$ can be found by applying Eqs. [5]-[9].
The dimensionless electrostatic interaction energy, $V\left(h_{1}\right.$ $\left.+h_{2}\right)$, is calculated by

$$
\begin{aligned}
V\left(h_{1}+h_{2}\right) & =f\left(h_{1}+h_{2}\right)-f(\infty) \\
& =\frac{1}{2} \int_{V_{1}} \rho_{1}^{*} y_{2} d V_{1}+\frac{1}{2} \int_{V_{2}} \rho_{2}^{*} y_{1} d V_{2},
\end{aligned}
$$

where $V_{i}$ is the volume of particle $i$, and $f\left(h_{1}+h_{2}\right)$ is the Helmholtz free energy when the center-to-center distance between two particles is $\left(h_{1}+h_{2}\right)$. The value of $f$ is calculated by employing the method suggested by Verwey and Overbeek (21).

\section{RESULTS AND DISCUSSION}

Figures 4 and 5 show the simulated dimensionless electrical potential distribution, defined as $\left(y / c_{1}^{2} \rho_{1}^{*}\right)$, for two values of $\kappa$. A comparison between Figs. 4 and 5 reveals that, for a fixed particle size, the greater the $\kappa$ (the greater the ionic strength or the thinner the electrical double layer), the lower the electrical potential inside a particle. This is because that the greater the ion strength, the greater the shielding effect (2). On the other hand, for a fixed $\kappa$, the larger the particle, the higher the electrical potential.

The variations in the dimensionless interaction free energy between two particles as a function of the relative orientation between them are summarized in Tables 1 and 2. Since spheroids are axisymmetric, only the domain defined by 0 

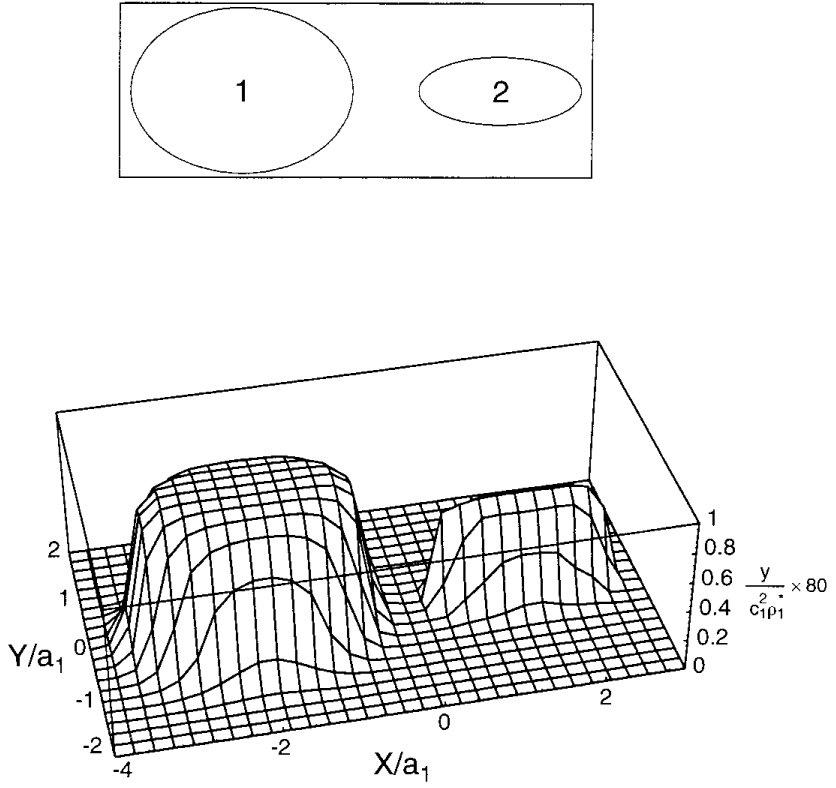

FIG. 4. Simulated dimensionless electrical potential distribution for the case $c_{1}^{2}=c_{2}^{2}=80, \rho_{1}=\rho_{2}, a_{1}=a_{2}, h_{1}=2, h_{2}=1.5, \xi_{S i}=1.5$, and $\xi_{S i}$ $=1.1$.

$\leqslant q_{1} \leqslant p, 0 \leqslant f_{1} \leqslant p / 2,0 \leqslant q_{2} \leqslant p / 2$, and $f_{2}=0$ is examined. Table 1 reveals that the interaction free energy is the greatest if the major axes of two particles lie on the same line (head-to-head, $q_{1}=0, f_{1}=0, q_{2}=0$, and $f_{2}=0$ ), and the smallest if their major axes are perpendicular to each other but not on the same plane (perpendicular, $q_{1}=0, f_{1}$ $=p / 2, q_{2}=p / 2$, and $\left.f_{2}=0\right)$. As shown in Table 2 , this is also true if the sizes of two particles are different. As indicated by Eq. [14], the interaction energy between two particles depends on the separation distance between the volume element of a particle and that of the other particle $\left(y_{i}\right.$ is a function of separation distance). In other words, the mean separation distance between two particles plays a significant role in the determination of their interaction energy. For a fixed center-to-center distance, two particles have the minimum of the minimal surface-to-surface distance if they are head-to-head. However, they also have the maximum of the maximal surface-to-surface distance. The net effect is that the interaction energy between two particles is the greatest if they are at a head-to-head position. On the other hand, the mean distance between two particles is the shortest if they are perpendicular to each other, which leads to the smallest interaction energy.

\section{Special Cases}

Several results reported in the literature can be recovered as the special cases of the general problem considered in the present study.
Particles covered by an ion-penetrable membrane. It is known that if a membrane containing fixed charges in an electrolyte solution is sufficiently thick, the electrical potential deep inside will reach a constant value, the Donnan potential (2). For the present case, if a particle is large enough, or the concentration of electrolyte is sufficiently high, the dimensionless Donnan potential for particle $i$, $y_{i, \mathrm{DON}}$, is

$$
y_{i, \mathrm{DON}}=\frac{4 \pi e \rho_{i}}{\varepsilon k_{\mathrm{B}} T \kappa^{2}}=\rho_{i}^{*}, \quad i=1,2 .
$$

This can also be obtained by resorting to Eqs. [2] and [3]. Since the net charges must vanish deep inside a particle, both the first and the second derivatives of the electrical potential with respect to position variable vanish. This implies that the present analysis is applicable to the case a particle composed of a rigid, uncharged core and an ionpenetrable membrane bearing fixed charges, as long as the latter is sufficiently thick compared to the double layer. In the case of Fig. 4, the left particle is large enough so that the electrical potential reaches its Donnan potential for -2.9 $\leqslant X_{1} / a_{1} \leqslant-1.1$. The particle on the right is too small to reach its Donnan potential. Similarly, in the case of Fig. 5, the value of $\kappa$ is too small for both particles to reach their Donnan potentials.

Two ion-penetrable spheres. If $\xi_{\mathrm{s}}$ is sufficiently large, Eqs. [7a]-[7c] yield
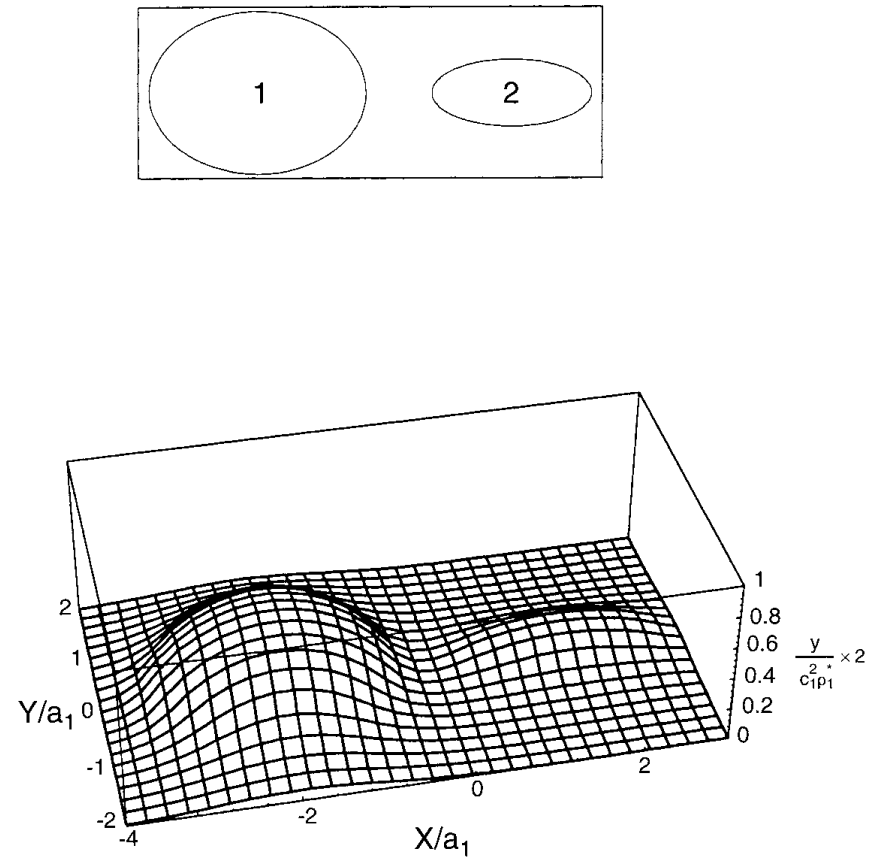

FIG. 5. Simulated dimensionless electrical potential distribution for the case described in the legend to Fig. 4, except that $c_{1}^{2}=c_{2}^{2}=2$. 


\section{TABLE 1}

Variation in the D imensionless Interaction Free E nergy between Two Spheroidal Particles, $V / \rho^{*}$, as a Function of the R elative Orientation between Them for the Case $\phi_{2}=0, h_{1}=h_{2}=1.2, c_{1}^{2}=c_{2}^{2}=0.5, \rho_{1}^{*}=\rho_{2}^{*}=\rho^{*}$, and $\xi_{1}=\xi_{2}=1.1$

\begin{tabular}{|c|c|c|c|c|c|c|c|c|c|c|c|c|c|c|c|c|c|c|c|}
\hline$\theta_{2}$ & 0 & 0 & 0 & & & & & $\pi / 4$ & & & & & & & & $\pi / 2$ & & & \\
\hline$\theta_{1}$ & 0 & 0 & 0 & 0 & $\pi / 4$ & $\pi / 2$ & $3 \pi / 2$ & 0 & $\pi / 4$ & $\pi / 2$ & $3 \pi / 2$ & 0 & 0 & $\pi / 4$ & $\pi / 2$ & 0 & $\pi / 4$ & $\pi / 2$ & 0 \\
\hline $\begin{array}{l}\phi_{1} \\
V I \rho^{*}\end{array}$ & 0 & $\pi / 4$ & $\pi / 2$ & 0 & 0 & 0 & 0 & $\pi / 4$ & $\pi / 4$ & $\pi / 4$ & $\pi / 4$ & $\pi / 2$ & 0 & 0 & 0 & $\pi / 4$ & $\pi / 4$ & $\pi / 4$ & $\pi / 2$ \\
\hline$\times 100$ & 4.594 & 3.894 & 3.419 & 3.894 & 3.306 & 3.001 & 3.469 & 3.312 & 3.130 & 2.990 & 3.196 & 2.975 & 3.419 & 2.998 & 2.666 & 2.974 & 2.804 & 2.654 & 2.640 \\
\hline
\end{tabular}

$$
X_{i} \approx Y_{i} \approx Z_{i}
$$

This means that a particle can be treated as a sphere. In this case, Eqs. [13a]-[13c] lead to the solution for spherical particles.

An ion-penetrable spheroid and a rigid plate. By referring to Fig. 6, we consider the interaction between an ionpenetrable spheroid and a rigid, charged plate located on the $Y-Z$ plane. In this case the method of images (3) can be applied. Let the original particle be particle 1 , and its image against the $Y-Z$ plane be particle 2 . We have $\theta_{1}=-\theta_{2}, \phi_{1}$ $=-\phi_{2}$, and $\xi_{\mathrm{S} 1}=\xi_{\mathrm{S} 2}$. If the rigid plate remains at constant potential, we choose $\rho_{2}^{*}=-\rho_{1}^{*}$. In this case the solution to Eq. [1] is

$$
\begin{aligned}
y= & \rho_{1}^{*}+\sum_{m=0}^{\infty} B_{2 m}\left(c_{1}\right) \\
& \times\left(R_{2 m}^{(1)}\left(c_{1}, \xi_{1}\right)-\frac{R_{2 m}^{\prime(1)}\left(c_{1}, 1\right)}{R_{2 m}^{\prime(2)}\left(c_{1}, 1\right)} R_{2 m}^{(2)}\left(c_{1}, \xi_{1}\right)\right) \\
& \times S_{2 m}\left(c_{1}, \eta_{1}\right)-\sum_{m=0}^{\infty} A_{2 m}\left(c_{1}\right) R_{2 m}^{(2)}\left(c_{1}, \xi_{2}\right) S_{2 m}\left(c_{1}, \eta_{2}\right) \\
& +y_{\mathrm{p}} \exp (\kappa x), 1 \leqslant \xi_{1} \leqslant \xi_{\mathrm{s} 1}, \\
y= & \sum_{m=0}^{\infty} A_{2 m}\left(c_{1}\right)\left[R_{2 m}^{(2)}\left(c_{1}, \xi_{1}\right) S_{2 m}\left(c_{1}, \eta_{1}\right)\right. \\
& \left.-R_{2 m}^{(2)}\left(c_{1}, \xi_{2}\right) S_{2 m}\left(c_{1}, \eta_{2}\right)\right] \\
& +y_{\mathrm{p}} \exp (\kappa x), \xi_{1} \geqslant \xi_{\mathrm{s} 1}, x \leqslant 0,
\end{aligned}
$$

where $y_{\mathrm{p}}$ is the dimensionless surface potential of the plate. On the other hand, if the plate remains at constant surface charge, then we choose $\rho_{2}^{*}=\rho_{1}^{*}$. The solution to Eq. [1] becomes

$$
\begin{aligned}
y= & \rho_{1}^{*}+\sum_{m=0}^{\infty} B_{2 m}\left(c_{1}\right) \\
& \times\left(R_{2 m}^{(1)}\left(c_{1}, \xi_{1}\right)-\frac{R_{2 m}^{\prime(1)}\left(c_{1}, 1\right)}{R_{2 m}^{\prime(2)}\left(c_{1}, 1\right)} R_{2 m}^{(2)}\left(c_{1}, \xi_{1}\right)\right) \\
& \times S_{2 m}\left(c_{1}, \eta_{1}\right)+\sum_{m=0}^{\infty} A_{2 m}\left(c_{1}\right) R_{2 m}^{(2)}\left(c_{1}, \xi_{2}\right) S_{2 m}\left(c_{1}, \eta_{2}\right) \\
& +\sigma^{\prime} \exp (\kappa x), 1 \leqslant \xi_{1} \leqslant \xi_{\mathrm{s} 1} \\
y= & \sum_{m=0}^{\infty} A_{2 m}\left(c_{1}\right)\left[R_{2 m}^{(2)}\left(c_{1}, \xi_{1}\right) S_{2 m}\left(c_{1}, \eta_{1}\right)\right. \\
& \left.+R_{2 m}^{(2)}\left(c_{1}, \xi_{2}\right) S_{2 m}\left(c_{1}, \eta_{2}\right)\right] \\
& +\sigma^{\prime} \exp (\kappa x), \xi_{1} \geqslant \xi_{\mathrm{s} 1}, x \leqslant 0
\end{aligned}
$$

\begin{tabular}{|c|c|c|c|c|c|c|c|c|c|c|c|c|c|c|c|c|c|c|c|}
\hline$\theta_{2}$ & 0 & 0 & 0 & & & & & $\pi / 4$ & & & & & & & & $\pi / 2$ & & & \\
\hline$\theta_{1}$ & 0 & 0 & 0 & 0 & $\pi / 4$ & $\pi / 2$ & $3 \pi / 2$ & 0 & $\pi / 4$ & $\pi / 2$ & $3 \pi / 2$ & 0 & 0 & $\pi / 4$ & $\pi / 2$ & 0 & $\pi / 4$ & $\pi / 2$ & 0 \\
\hline $\begin{array}{l}\phi_{1} \\
V / \rho^{*}\end{array}$ & 0 & $\pi / 4$ & $\pi / 2$ & 0 & 0 & 0 & 0 & $\pi / 4$ & $\pi / 4$ & $\pi / 4$ & $\pi / 4$ & $\pi / 2$ & 0 & 0 & 0 & $\pi / 4$ & $\pi / 4$ & $\pi / 4$ & $\pi / 2$ \\
\hline$\times 100$ & 6.691 & 5.817 & 5.193 & 5.634 & 4.881 & 4.487 & 5.102 & 4.958 & 4.653 & 4.471 & 4.745 & 4.448 & 4.886 & 4.355 & 3.927 & 4.325 & 4.104 & 3.909 & 3.888 \\
\hline
\end{tabular}

where $\sigma^{\prime}$ is the dimensionless surface charge density of the plate defined by

$$
\sigma^{\prime}=\frac{4 \pi e \sigma}{\varepsilon \kappa k_{\mathrm{B}} T},
$$

TABLE 2

Variation in the D imensionless Interaction Free E nergy between Two Spheroidal Particles, $V / \rho^{*}$, as a Function of the R elative Orientation between Them for the C ase $\phi_{2}=0, h_{1}=1.2, h_{2}=1.35, c_{1}^{2}=c_{2}^{2}=0.5, \rho_{1}^{*}=\rho_{\mathbf{c}}^{*}=\rho^{*}, \xi_{1}=1.1$, and $\xi_{2}=1.25$ 


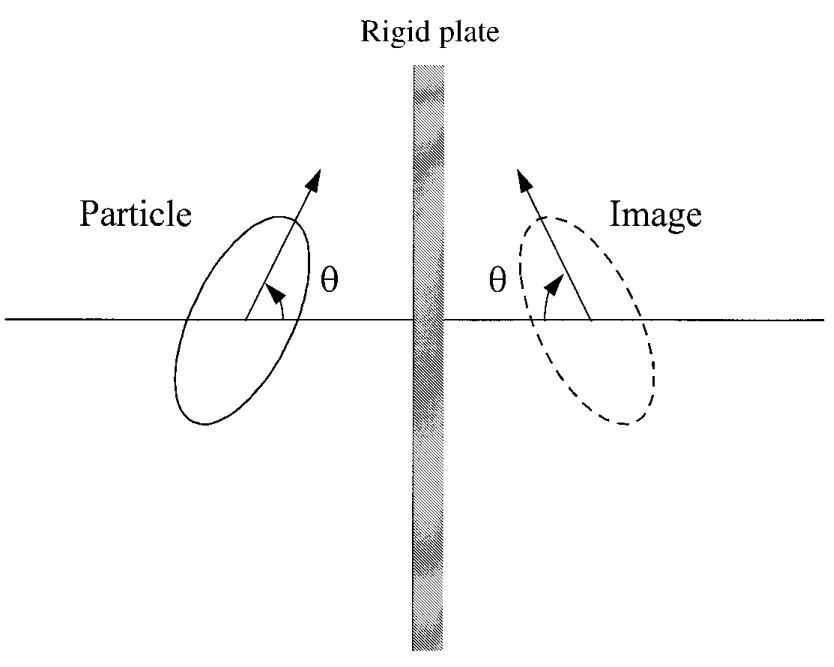

Y-Z plane

FIG. 6. A schematic representation for the case of an ion-penetrable spheroid and a rigid plate.

If we denote $S_{\mathrm{p}}$ as the area of the plate, the dimensionless interaction free energy is

$$
\begin{aligned}
V\left(h_{1}\right)=\frac{1}{2} \int_{V_{1}} \rho_{1}^{*} \cdot\left(y_{2}+y_{\mathrm{p}}\right. & \exp (\kappa x)) d V_{1} \\
& \pm \frac{1}{2} \int_{S_{\mathrm{p}}} \sigma^{\prime} \cdot\left(y_{1}+y_{2}\right) d S
\end{aligned}
$$

where the negative sign is for the case of constant potential and the positive sign for constant charge density.

\section{APPE NDIX}

Under the Debye-Huckle condition, the Poisson-Boltzmann equation for spheroidal surfaces can be approximated by

$$
\begin{array}{r}
\frac{\partial}{\partial \eta}\left[\left(1-\eta^{2}\right) \frac{\partial y}{\partial \eta}\right]+\frac{\partial}{\partial \xi}\left[\left(\xi^{2}-j\right) \frac{\partial y}{\partial \xi}\right] \\
+\frac{\xi^{2}-j \eta^{2}}{\left(\xi^{2}-j\right)\left(1-\eta^{2}\right)} \frac{\partial^{2} y}{\partial \Phi^{2}}-c^{2}\left(\xi^{2}-j \eta^{2}\right) y \\
=0,(1+j) / 2 \leqslant \xi \leqslant \infty,-1 \leqslant \eta \leqslant 1, \\
0 \leqslant \Phi \leqslant 2 \pi, \quad[\text { [A } 1]
\end{array}
$$

where $(\xi, \eta, \Phi)$ denotes the spheroidal coordinates, $j$ is an index parameter $(j=1$ for prolate spheroid, $j=-1$ for oblate spheroid), and $c=\kappa a, a$ being the distance between the focus and the center of a spheroid. The solution to this equation can be expressed as

$$
y=\sum R_{m n}(\xi) S_{m n}(\eta) \exp (i m \Phi),
$$

where $S_{m n}$ and $R_{m n}$ are the angle and the radial functions (22), respectively. $S_{m n}$ is defined by

$$
S_{m n}(\eta)=\sum_{s=0,1}^{\infty} A_{m n, s} P_{m+s}^{m}(\eta),
$$

where $s$ is even if $(n-m)$ is even, and odd if $(n-m)$ is odd, $P_{n}^{m}$ is the associated Legendre function of the first kind of order $m$ with degree $n$. The coefficient $A_{m n, s}$ in Eq. [A3] satisfies the recursive relation

$$
\begin{array}{r}
j c^{2} \cdot \alpha(s) \cdot A_{m n, s+2}+\left[-(m+s)(m+s+1)+\lambda_{m n}\left(j c^{2}\right)\right. \\
\left.+j c^{2} \cdot \beta(s)\right] \cdot A_{m n, s}+j c^{2} \cdot \gamma(s) \cdot A_{m n, s-2}=0, \quad[\mathrm{~A} 4 \mathrm{a}
\end{array}
$$

where

$$
\begin{aligned}
& \alpha(s)=\frac{(s+2 m+2)(s+2 m+1)}{(2 s+2 m+5)(2 s+2 m+3)} \\
& \beta(s)=\frac{2 s^{2}+4 s m+2 s+2 m-1}{(2 s+2 m-1)(2 s+2 m+3)} \\
& \gamma(s)=\frac{s(s-1)}{(2 s+2 m-3)(2 s+2 m-1)} .
\end{aligned}
$$

To determine the coefficient $A_{m n, s}$ in [A4a], the eigenvalue $\lambda_{m n}$ needs to be estimated first. This can be done through a trial-and-error procedure based on the condition that $\left(A_{m n, s+2} / A_{m n, s}\right)$ must vanish as $s$ approaches infinity. This is tedious and time consuming, in general, and another approach is suggested. If $c$ is sufficiently small, $A_{m n, s}$ and $\lambda_{m n}$ can be expanded as

$$
\begin{aligned}
A_{m n, s} & =\sum_{r=0}^{\infty} a_{m n, r, s}\left(j c^{2}\right)^{r} \\
\lambda_{m n} & =\sum_{r=0}^{\infty} a_{m n, r}\left(j c^{2}\right)^{r} .
\end{aligned}
$$

$\lambda_{m n, r}$ and $a_{m n, r, s}$ can be calculated on the basis of the recursive relations

$$
\begin{aligned}
& \lambda_{m n, r}=-\left[\alpha(n-m) a_{m n, r-1, n-m+2}\right. \\
& \quad+\beta(n-m) a_{m n, r-1, n-m}+\gamma(n-m) a_{m n, r-1, n-m-2}
\end{aligned}
$$




$$
\begin{aligned}
& a_{m n, r, s}=\frac{1}{(s+m)(s+m+1)-\lambda_{m n, 0}} \\
& \times\left\{\begin{array}{c}
\alpha(s) a_{m n, r-1, s+2}+\beta(s) a_{m n, r-1, s}+\gamma(s) a_{m n, r-1, s-2} \\
+\sum_{p=1}^{r-1} \lambda_{m n, p} \cdot a_{m n, r-p, s}
\end{array}\right\} \\
& s \neq n \quad[\mathrm{~A} 6 \mathrm{~b}] \\
& a_{m n, r, s}=0, \quad s=n .
\end{aligned}
$$

The radial functions of the first and the second kinds can be expressed, respectively, as $(23,24)$

$$
\begin{aligned}
& R_{m n}^{(1)}(c, \xi) \\
& \quad=\sum_{s=0,1}^{\infty} A_{m n, s}\left(\frac{j \xi^{2}-1}{\xi^{2}}\right)^{m / 2} \frac{(2 m+s) !}{s !} I_{m+s}(c \xi)
\end{aligned}
$$

and

$$
\begin{aligned}
& R_{m n}^{(2)}(c, \xi) \\
& \quad=\sum_{s=0,1}^{\infty} A_{m n, s}\left(\frac{j \xi^{2}-1}{\xi^{2}}\right)^{m / 2} \frac{(2 m+s) !}{s !} K_{m+s}(c \xi),
\end{aligned}
$$

where $I_{m+s}$ and $K_{m+s}$ are, respectively, the modified spherical Bessel functions of the first and the third kinds of order $(m$ $+s)$. We have

$$
\begin{aligned}
I_{n} & =\sqrt{\frac{\pi}{2 x}} i_{n+1 / 2} \\
K_{n} & =\sqrt{\frac{\pi}{2 x}} k_{n+1 / 2},
\end{aligned}
$$

where $i_{n+1 / 2}$ and $k_{n+1 / 2}$ are the modified Bessel functions of the first and the second kinds, respectively. Note that if $c \xi$ is small, $K_{n}$ diverges, so does $R_{m n}^{(2)}$. In this case, $R_{m n}^{(2)}$ needs to be replaced by another expression. Aoi (25) suggested using

$$
\begin{gathered}
R_{m(m+2 s)}^{(2)}(c, \xi)=\left(\xi^{2}-j\right)^{m / 2} \sum_{q=0}^{\infty} \omega_{2 q} K_{m+q}\left[\frac{c}{2}\left(\xi+\sqrt{\xi^{2}-j}\right)\right] \cdot I_{m+q}\left[\frac{j c}{2}\left(\xi-\sqrt{\xi^{2}-j}\right)\right], \\
R_{m(m+2 s+1)}^{(2)}(c, \xi)=\xi\left(\xi^{2}-j\right)^{m / 2} \sum_{q=0}^{\infty} \omega_{2 q+1} K_{m+q+1}\left[\frac{c}{2}\left(\xi+\sqrt{\xi^{2}-j}\right)\right] \times I_{m+q+1}\left[\frac{j c}{2}\left(\xi-\sqrt{\xi^{2}-j}\right)\right],
\end{gathered}
$$

where

$$
\omega_{2 q}=(2 m+2 q+1) \times \sum_{s=0}^{q} \sum_{t=s}^{q}(-1)^{t+[(1-j) q / 2]} \frac{2^{m+2 s+1}(2 m+q+t) !(2 m+2 s) !(2 t) !(t+m+s) !}{t !(m+t) !(q-t) !(2 s) !(t-s) !(2 t+2 m+2 s+1) !} A_{m n, 2 s}
$$

[A8c ]

$$
\begin{aligned}
\omega_{2 q}=(2 m+2 q+3) \times \sum_{s=0}^{q} & \sum_{t=s}^{q}(-1)^{t+[(1-j) q / 2]} \\
& \quad \frac{2^{m+2 s+2}(2 m+q+t+2) !(2 m+2 s+1) !(2 t+1) !(t+m+s+1) !}{t !(m+t+1) !(q-t) !(2 s+1) !(t-s) !(2 t+2 m+2 s+3) !} A_{m n, 2 s+1}
\end{aligned}
$$

For an axisymmetric problem, Eqs. [A3], [A7a], [A7b], [A8a], and [A8b] with $m=0$ should be employed, and $S_{m n}, R_{m n}^{(1)}$, and $R_{m n}^{(2)}$ reduce to $S_{n}, R_{n}^{(1)}$, and $R_{n}^{(2)}$, respectively.

\section{ACK NOWLEDGMENT}

This work was supported by the National Science Council of the Republic of China.

\section{REFERENCES}

1. Hunter, R. J., in "Foundations of Colloid Science." Vol. 1, Oxford Univ. Press, London, 1989.

2. Ohshima, H., and Kondo, T., J. Colloid Interface Sci. 155, 499 (1993).

3. Ohshima, H., and Kondo, T., J. Colloid Interface Sci. 157, 504 (1993).

4. Ohshima, H., J. Colloid Interface Sci. 162, 487 (1994).

5. Ohshima, H., J. Colloid Interface Sci. 168, 255 (1994).

6. Ohshima, H., Adv. Colloid Interface Sci. 53, 77 (1994).

7. Ohshima, H., J. Colloid Interface Sci. 170, 432 (1995).

8. Ohshima, H., J. Colloid Interface Sci. 176, 7 (1995).

9. Ohshima, H., and Kondo, T., Colloid Polym. Sci. 271, 1191 (1993). 
10. Krozel, J. W., and Saville, D. A., J. Colloid Interface Sci. 150, 365 (1992).

11. Sengupta, A. K., and Papadopoulos, K. D., J. Colloid Interface Sci. 149, 135 (1992).

12. Allain, C., and Cloitre, M., J. Colloid Interface Sci. 157, 261 (1993).

13. Anandarajah, A., and Chen, J., J. Colloid Interface Sci. 168, 111 (1994).

14. Israelachvili, J. N., in "Intermolecular and Surface Forces.' Academic Press, San Diego, 1985.

15. Fisher, L., J. Chem. Soc. Faraday Trans. 89, 2567 (1993).

16. Hsu, J. P., Lin, D. P., and Tseng, S. J., Colloid Polym. Sci. 273, 271 (1995).

17. Tortora, G. J., Funke, B. R., and Case, C. L., in "Microbiology: An Introduction,'” 4th ed. Benjamin-Cummings, Redwood City, CA 1992.
18. Meirovitch, L., in "'Methods of Analytical Dynamics." McGraw-Hill, New York, 1994.

19. Sokolnikoff, I. S., in "Tensor Analysis: Theory and Applications to Geometry and Mechanics of Continua,' 2nd ed. Wiley, New York, 1964.

20. Moon, P., and Spencer, D. E., in "Field Theory Handbook.' SpringerVerlag, Berlin, 1961.

21. Verwey, E. J. W., and Overbeek, J. Th. G., in "'Theory of the Stability of Lyophobic Colloids.' Elsevier, Amsterdam, 1948.

22. Flammer, C., in "Spheroidal Wave Functions." Stanford Univ. Press, Stanford, CA, 1957.

23. Hsu, J. P., and Liu, B. T., J. Colloid Interface Sci. 183, 214 (1996).

24. Yoon, B. J., and Kim, S., J. Colloid Interface Sci. 128, 275 (1989).

25. Aoi, T., J. Phys. Soc. Jpn. 10, 130 (1955). 